\title{
STABLE RANK OF THE DISC ALGEBRA
}

\author{
P. W. JONES, D. MARSHALL AND T. WOLFF ${ }^{1}$
}

ABstract. We prove that the Bass stable rank of the disc algebra is one.

Let $A$ be the disc algebra, consisting of functions analytic on the unit disc in $\mathbf{C}$ and continuous on its closure. We prove the following result:

THEOREM 1. Suppose $f_{1}, f_{2} \in A$ and $\left|f_{1}(z)\right|+\left|f_{2}(z)\right|>0$ for all $z$ with $|z| \leq 1$. Then there are $g_{1}, g_{2} \in A$ with $g_{1}^{-1} \in A$ and $g_{1} f_{1}+g_{2} f_{2}=1$.

The question of whether Theorem 1 is true arose in recent work of Rieffel [2] on $K$-theory of $C^{*}$-algebras. The connection is that Theorem 1 amounts to a computation of the Bass stable rank of $A$. If $R$ is any ring, then its Bass stable rank is by definition $\operatorname{bsr}(R)=\min \left(n\right.$ : whenever $r_{1} \cdots r_{n+1} \in R$ and $\left\{r_{j}\right\}$ generate $R$ as a left ideal, there are $b_{1} \cdots b_{n} \in R$ such that $r_{1}+b_{1} r_{n+1} \cdots r_{n}+b_{n} r_{n+1}$ generate $R$ as a left ideal). Since functions which generate $A$ cannot all vanish at the same point, one obtains $\operatorname{bsr}(A)=1$ by dividing the conclusion of Theorem 1 by $g_{1}$. Now in [2], Rieffel introduces another concept, the topological stable $\operatorname{rank}, \operatorname{tsr}(R)$. It is defined when $R$ is a Banach algebra and is $\operatorname{tsr}(R)=\min \left(n\right.$ : whenever $r_{1} \cdots r_{n} \in R$ and $\delta>0$ there are $b_{1} \cdots b_{n} \in R$ such that $\left\{b_{j}\right\}$ generate $R$ as a left ideal and $\left\|b_{j}-r_{j}\right\|<\delta$ ). Rieffel leaves open whether $\operatorname{tsr}(R)=\operatorname{bsr}(R)$ for all Banach algebras $R$, but suggests that the disc algebra should provide a counterexample. Theorem 1 shows that it does since, as he points out, it is easily seen that $\operatorname{tsr}(A)=2$.

Theorem 1 may be regarded as a variant of the Carleson corona theorem, which states that if $f_{1} \cdots f_{n}$ are bounded analytic functions on $D=\{z \in \mathbf{C :}|z|<1\}$ and $\Sigma\left|f_{j}(z)\right|>\delta>0$ on $D$, then there are bounded analytic functions $g_{1} \cdots g_{n}$ such that $\Sigma g_{j} f_{j}=1$. Our proof of Theorem 1 is in some sense a modification of the proof of the corona theorem given in [1], although the assumption of continuity at the boundary eliminates the analytic difficulties in the argument. The corona theorem itself can be proved quickly by soft techniques when the functions $f_{1} \cdots f_{n}$ are continuous up to the boundary (see [3, p. 396]), since one can identify the maximal ideal space of $A$ with $\bar{D}$. However, this argument does not give $g_{1}^{-1} \in A$. We make use of the following

Claim. Suppose $f_{1}$ and $f_{2}$ are as in Theorem 1. Then there is $\delta>0$ and a continuous function $F: \bar{D} \rightarrow \mathbf{C}$, Lipschitz on compact subsets of $D$, and such that

(1) $F(z)=f_{1}(z)$ if $\left|f_{1}(z)\right|<\delta, F(z)=1$ if $\left|f_{2}(z)\right|<\delta$,

(2) $|F(z)| \geq \delta$ if $\left|f_{1}(z)\right| \geq \delta$,

(3) $\partial F / \partial \bar{z}$ is bounded on $D$.

To construct $F$ choose closed sets $E_{1}$ and $E_{2}$ such that $E_{j}$ has finitely many components and $\left\{\left|f_{j}\right|<\delta\right\} \subseteq E_{j} \subseteq\left\{\left|f_{j}\right|<2 \delta\right\}$, where $\delta$ is small enough so that

Received by the editors February 3, 1983 and, in revised form, January 8, 1985.

1980 Mathematics Subject Classification. Primary 46J15; Secondary 30D50, 46L99.

${ }^{1}$ The authors were partially supported by the NSF. 
$\left\{\left|f_{1}\right|<2 \delta\right\} \cap\left\{\left|f_{2}\right|<2 \delta\right\}=\varnothing$. By the maximum principle, $E_{2}$ cannot separate any point of $E_{1}$ from the boundary of the disc. So we can extend the components of $E_{1}$ to the boundary to obtain a closed set $S \subseteq \bar{D}$ having the following properties: $S \supseteq E_{1}, S \cap E_{2}=\varnothing$, and $S$ has finitely many components each of which intersects $\partial D$. The components of $\bar{D} \backslash S$ are then simply connected, so a bounded continuous branch of $\log f_{1}$ exists on $\bar{D} \backslash S$. Since $E_{2}$ and $S$ are compact and $E_{2} \cap S=\varnothing$, there is a function $q \in C^{\infty}\left(R^{2}\right)$ such that $q=1$ on a neighborhood of $S$ and $q=0$ on a neighborhood of $E_{2}$. Define $F(z)=\exp \left(q(z) \log f_{1}(z)\right)$. Clearly $F$ is continuous on $\bar{D}$ and satisfies (1) and (2). As for (3) we have $\partial F / \partial \bar{z}=F \log f_{1}(\partial q / \partial \bar{z})$ when $z \notin S$ and zero otherwise, and $q$ is smooth. The claim is proved.

To prove Theorem 1 let $g_{1}=\left(F / f_{1}\right) \exp \left(u f_{2}\right)$, where $u$ is as yet unknown. For $g_{1}$ to belong to $A$ we need $u$ continuous on $\bar{D}$ and satisfying

$$
\frac{\partial u}{\partial \bar{z}}=\frac{1}{F f_{2}} \frac{\partial F}{\partial \bar{z}}
$$

on $D$. Set

$$
k=\frac{1}{F f_{2}} \frac{\partial F}{\partial \bar{z}} .
$$

By (1) and (2), $\left|F f_{2}\right| \geq \delta^{2}$ if $\partial F / \partial \bar{z} \neq 0$. So $k$ is bounded on $D$ and since the convolution of a bounded function and an $L^{1}$ function is continuous,

$$
u(z)=\frac{1}{\pi} \iint_{D} \frac{k(\zeta)}{\zeta-z} d \zeta d \bar{\zeta}
$$

has the desired properties.

Moreover, $g_{1}$ is bounded away from zero on $D$, so $g_{1}^{-1} \in A$. Let $g_{2}$ be determined by the condition $g_{1} f_{1}+g_{2} f_{2}=1$. Clearly, $g_{2}$ is continuous when $f_{2} \neq 0$. On the other hand, if $0<\left|f_{2}\right|<\delta$, then

$$
g_{2}=\frac{1}{f_{2}}\left(1-F \exp \left(u f_{2}\right)\right)=\frac{1}{f_{2}}\left(1-\exp \left(u f_{2}\right)\right)
$$

by (1), so $g_{2} \rightarrow-u$ as $f_{2} \rightarrow 0$. This finishes the proof.

We are grateful to Donald Sarason for communicating the problem.

\section{REFERENCES}

1. L. Carleson, The corona theorem, Proc. 15th Scandinavian Congress (Oslo, 1968), Lecture Notes in Math., vol. 118, Springer-Verlag, Berlin and New York, 1970.

2. M. Rieffel, Dimension and stable rank in the $K$-theory of $C^{*}$-algebras, Proc. London Math. Soc. (3) 46 (1983), 301-333.

3. W. Rudin, Real and complex analysis (2nd ed.), McGraw-Hill, New York, 1974.

Department of Mathematics, University of Chicago, Chicago, Illinois 60637

DEPARTMENT OF MATHEMATICS, UNIVERSiTy OF WASHington, SEATtLe, WASHINGTON 98195

Department of Mathematics, California institute of Technology, PasaDENA, CALIFORNIA 91125 